\title{
Regulation of Telomerase by Human Papillomaviruses
}

\author{
D.A. Galloway ${ }^{*}$ L.C. Gewin, ${ }^{* \dagger}$ H. Myers, ${ }^{*}$ W. Luo ${ }^{*}{ }^{\mathbb{I}}$ C. Grandori, ${ }^{*}$ \\ R.A. KatZENELlenbogen, ${ }^{*}$ AND J.K. MCDOUGALL* ${ }^{* \dagger}$ \\ *Program in Cancer Biology, Divisions of Human Biology and Public Health Sciences, \\ Fred Hutchinson Cancer Research Center, Seattle, Washington 98109-1024; ${ }^{\S}$ Department of Pediatrics, \\ University of Washington, Seattle, Washington 98195
}

\begin{abstract}
The E6 oncoprotein of human papillomaviruses (HPVs) induces telomerase activity in primary human epithelial cells. This activity is dependent on association of E6 with E6AP, a cellular ubiquitin ligase. E6 activates the transcription of $h T E R T$, the catalytic subunit of telomerase. E boxes near the start of $h T E R T$ transcription are required for E6; however, acetylated histones are only present in the E6 cells. We identified two isoforms of NFX1, a new binding partner of E6/E6AP. The NFX191 isoform binds to an X-box motif located adjacent to the proximal E box, binds Sin3A and HDACs, repressing $h T E R T$ transcription. It preferentially binds E6/E6AP and is targeted for ubiquitin-mediated degradation. The NFX1-123 isoform has the opposite activity, increasing $h T E R T$ transcription or translation. This is the first example of viral oncoproteins disrupting regulation of telomerase, a critical event in tumorigenesis.
\end{abstract}

Nearly $20 \%$ of cancers worldwide have a component of their etiology that is due to infectious agents. In some cases, infection has an indirect effect, such as the immunosuppression caused by HIV or the inflammation caused by Helicobacter pylori, but in other cases, such as human papillomaviruses (HPVs), viral gene products persist in the cancer and directly promote neoplasia. Understanding the mechanisms by which the viral genes disrupt the checkpoints that normally protect cells from cancer will likely provide insights into cancers in which the underlying critical abnormalities are more difficult to discern.

Both epidemiologic observations and molecular data firmly support a causal role for a group of HPVs in the etiology of virtually $100 \%$ of cervical carcinomas, as well as the majority of other anogenital cancers and a subset of head and neck cancers (Cogliano et al. 2005). Of these HPV types, HPV-16 DNA is found in more than $50 \%$ of tumors (Walboomers et al. 1999). Two viral genes, E6 and E7, are invariably retained and expressed in cervical cancers, and together E6 and E7 efficiently immortalize human epithelial cells. The E7 protein associates with the retinoblastoma $(\mathrm{Rb})$ family of proteins through a LXCXE motif and promotes the ubiquitin-mediated degradation of Rb, p107, and p130 (Munger et al. 2001). Degradation of $\mathrm{Rb}$ is necessary but not sufficient for E7's role in cellular immortalization, which also requires sequences in the carboxy-terminal zinc-like finger (Helt and Galloway 2001). HPV-16 E6 associates with a cellular protein, $\mathrm{E} 6 \mathrm{AP}$, and together the complex functions as a ubiquitin ligase (Huibregtse et al. 1993). The p53 tumor suppressor is the best-studied target of E6/E6AP, and its degradation eliminates several checkpoints that normally maintain ge-

\footnotetext{
"This work is dedicated to J.K.M., who died September 13, 2003, in recognition of his seminal contributions to the papillomavirus field.

Present addresses: ${ }^{\dagger}$ Salk Institute, La Jolla, California; ${ }^{*}$ University of California at San Francisco Medical School, San Francisco, California; "Vanderbilt University, Nashville, Tennessee.
}

netic stability (Kessis et al. 1993; Demers et al. 1994).

Disruption of the $\mathrm{Rb}$ and $\mathrm{p} 53$ pathways is critical for transformation of many human cell types, but it is also essential to prevent telomere shortening (Hahn et al. 1999). In some strains of human fibroblasts, the introduction of hTERT, the catalytic subunit of telomerase, is sufficient for immortalization (Bodnar et al. 1998; Kiyono et al. 1998; Vaziri and Benchimol 1998; Wang et al. 1998). Telomerase may play additional roles in tumorigenic transformation, because hTERT was necessary for transformation of cells that maintained long telomeres by the ALT pathway (Stewart et al. 2002). Nearly all tumors and cells transformed in culture express hTERT at levels that provide sufficient telomerase activity to keep telomeres above a critically short level (Kim et al. 1994). Multiple mechanisms are likely responsible for regulation of telomerase activity, including changes in transcription factors (Xu et al. 2001), loss of transcriptional repressors (Horikawa et al. 1998; Ducrest et al. 2001; Lin and Elledge 2003), changes in chromatin structure (Takakura et al. 2001; Hou et al. 2002), and altered levels of telomere-binding proteins (van Steensel et al. 1998). Additionally, cancers may arise in stem cells in which telomerase is constitutively active.

Immortalization of cells by oncogenes such as SV40 T antigen is accompanied by a stage known as crisis, or M2, in which there is both proliferation and apoptosis, and massive chromosomal instability resulting from cycles of fusion and breakage (Shay et al. 1991). A small population of cells emerge from crisis that have activated telomerase by unknown mechanisms. In contrast, immortalization of human epithelial cells by HPV E6 and E7 shows very little, if any, evidence of crisis (McDougall 1994). Although cells expressing E6/E7 show some telomere shortening, a significant proportion of cells in the culture express sufficient telomerase activity to proliferate indefinitely (Klingelhutz et al. 1994). This suggested that, unlike T antigen, the HPV oncoproteins might directly activate telomerase. 
Indeed, expression of HPV-16 E6, but not E7, was shown to induce telomerase activity (Klingelhutz et al. 1996). The effects of E6 were observed in two epithelial cell types, keratinocytes, and mammary epithelial cells, but not in fibroblasts (Klingelhutz et al. 1996; Wang et al. 1998). Using mutated E6 proteins, we showed that the induction of telomerase was distinct from the degradation of p53. Further studies indicated that E6 was able to activate transcription of hTERT (Gewin and Galloway 2001; Oh et al. 2001; Veldman et al. 2001). However, clones of E6-expressing cells had variable levels of telomerase activity, suggesting epigenetic influences on hTERT transcription. In this paper, we discuss what is known about the mechanism by which HPV-16 E6 activates hTERT transcription, and we speculate on a role for telomerase induction in the life cycle of HPVs.

\section{E6, c-Myc, AND hTERT TRANSCRIPTION}

The hTERT promoter contains many known transcription-factor-binding sites, including several $E$ boxes and Sp1 sites (Horikawa and Barrett 2003). Although c-Myc is able to bind the E boxes in the hTERT promoter and can induce $h T E R T$ expression in many cell types (Wang et al. 1998; Greenberg et al. 1999; Wu et al. 1999), we and other investigators have found no correlation between the level of c-Myc expression and the ability of E6 to induce hTERT (Gewin and Galloway 2001; Oh et al. 2001; Veldman et al. 2001). We have, however, determined that the promoter region including the proximal $\mathrm{E}$ box is important for E6-mediated hTERT induction (Gewin and Galloway 2001; Veldman et al. 2001). Mutations in the $\mathrm{E}$ boxes that disrupt c-Myc binding severely diminish the ability of either c-Myc or E6 to trans-activate the $h T E R T$ promoter.

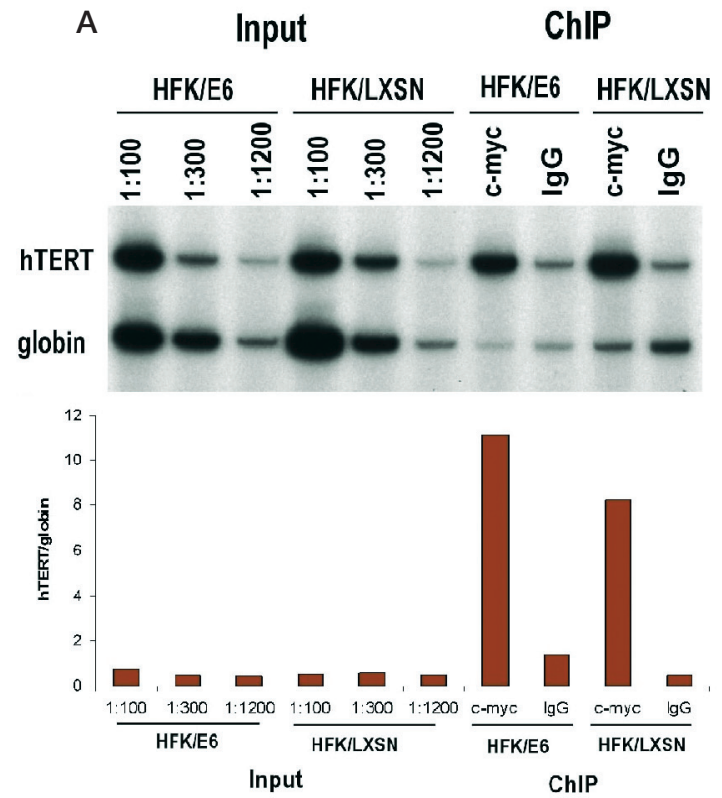

To determine whether c-Myc binding to the hTERT promoter was influenced by E6, chromatin immunopreciptation assays (ChIP) were performed on human foreskin keratinocytes (HFKs) expressing HPV-16 E6 or a control vector (LXSN) (Fig. 1). c-Myc occupied the hTERT promoter in E6 expressing HFKs in which hTERT was expressed, as well as in the LXSN-HFKs that had little or no hTERT expression. Although the overall levels of c-Myc were equivalent in the two cell types, the E6-HFKs reproducibly showed more c-Myc at the hTERT promoter than did the LXSN-HFKs, but never even a twofold difference, which was not enough to explain the apparent on-off switch of hTERT expression. Interestingly, when the hTERT promoter was interrogated for markers of transcriptionally active chromatin, such as acetylated histone $\mathrm{H} 3$ and $\mathrm{H} 4$, only the E6-HFKs showed these markers at the hTERT promoter (Fig. 1B). This indicated that although c-Myc was able to bind to the hTERT promoter, it was unable to recruit the necessary machinery, including histone acetyltransferases to activate $h T E R T$ transcription. The minimal hTERT promoter contains two c-Myc-binding sites, one that is $40 \mathrm{bp}$ downstream of the transcription start site and another that is 220 bp upstream of the start. We have not been able to determine whether c-Myc occupies one or both of these sites, or whether E6 changes the occupation. Although c-Myc steady-state levels do not change upon E6 expression, it is possible that this is a result of balanced c-Myc induction (Kinoshita et al. 1997) and increased degradation (Gross-Mesilaty et al. 1998). E6/E6AP has been demonstrated to ubiquitinate c-Myc in vitro and in vivo (Gross-Mesilaty et al. 1998). In fact, E6 has been found at the hTERT promoter, and E6 can immunoprecipitate cMyc from cell lysates (Veldman et al. 2003). Although we have been unable to demonstrate an interaction be-



Figure 1. ChIP of c-Myc and acetylated histones at the hTERT promoter. Lysates of HFKs expressing either vector (LXSN) or E6 were crosslinked and immunoprecipitated with antibodies to c-Myc $(A)$, acetylated histone $\mathrm{H} 3(B)$, or acetylated histone H4. After reversal of the crosslink, PCR was performed with primers spanning the two c-Myc-binding sites in the $h T E R T$ promoter, and to a region of human $\beta$-globin. A nonspecific IgG antibody was used as a control and the hTERT signal was normalized to a $\beta$-globin control. 
tween c-Myc and E6, it remains possible that these proteins do interact in a complex at the $h T E R T$ promoter.

Many of the activities of the high-risk E6 proteins require their association with E6AP and degradation of target proteins. When we used mutated E6 proteins to identify domains of E6 that were necessary for activation of hTERT, we noted that binding of E6AP was correlated with hTERT activation but was independent of binding to p53 (Kiyono et al. 1998; Gewin and Galloway 2001). To test for the requirement for E6AP activation of $h T E R T$ transcription, we knocked down the expression of E6AP using shRNAs (Fig. 2). Of three shRNAs to E6AP, Esh1 expression most efficiently restored the levels of p53 in E6-expressing cells (Fig. 2B). Similarly, Esh1 strongly reduced telomerase activity as measured in a telomerase repeat amplification protocol (TRAP) assay, and it reduced the level of $h T E R T$ RNA. Furthermore, knockdown of E6AP in E6-expressing cells reduced the levels of acetylated histones $\mathrm{H} 3$ and $\mathrm{H} 4$. These data indicate that E6AP is required for the ability of E6 to activate $h T E R T$ transcription. We have not been able to show that the ubiquitin ligase activity of E6AP is required for $h T E R T$ transcription by expressing a catalytically dead form of E6AP (E6APC833A) (L. Gewin, unpubl.); however, the endogenous E6AP may be recruited to E6/E6AP-C833A complexes.

\section{NFX1-91 IS A NOVEL REPRESSOR OF hTERT TRANSCRIPTION}

Finding that E6AP was required for E6 activation of $h T E R T$ transcription prompted us to look for novel targets of E6/E6AP that might repress $h T E R T$ transcription. A survey of previously identified cellular targets of E6/E6AP did not reveal any logical contenders for hTERT transcriptional regulators; therefore, we began to search for new targets of E6/E6AP by a yeast two-hybrid screen. The bait construct consisted of E6AP-C833A fused to the Gal4 DNA-binding domain. To ensure equal dosage of both E6 and E6AP, the plasmid also encoded the E6 oncoprotein. In a screen of both fetal brain and HeLa cell cDNA libraries, several known E6/E6AP interactors were identified. In addition, a new E6/E6AP target protein identified in the screen was NFX1 (nuclear factor binds to the $X 1$ box), a transcriptional repressor of MHC class II genes (Song et al. 1994). NFX1 was originally described and cloned in a screen for proteins that bound to the X-box region of MHC class II genes and is thought to be involved in a feedback loop to limit the immune response following infection.

Recent advances in the annotation of the human genome revealed that $N F X 1$ has two splice variants encoding isoforms with identical amino termini and variant carboxyl termini (Fig. 3, Unigene Hs. 413074). We will specify the longer 1120 -amino acid isoform here as NFX1-123, because it is approximately $123 \mathrm{kD}$, and the shorter 833-amino acid isoform as NFX1-91 ( 91 kD). We demonstrated that both isoforms of NFX1 are expressed in HFKs. Both isoforms have a RING finger/ PHD finger domain; found in many E3 ubiquitin ligases, this domain has been shown to confer autoubiquitination activity to NFX1 in in vitro assays (Lorick et al. 1999). The RING finger domain is followed by several cys-

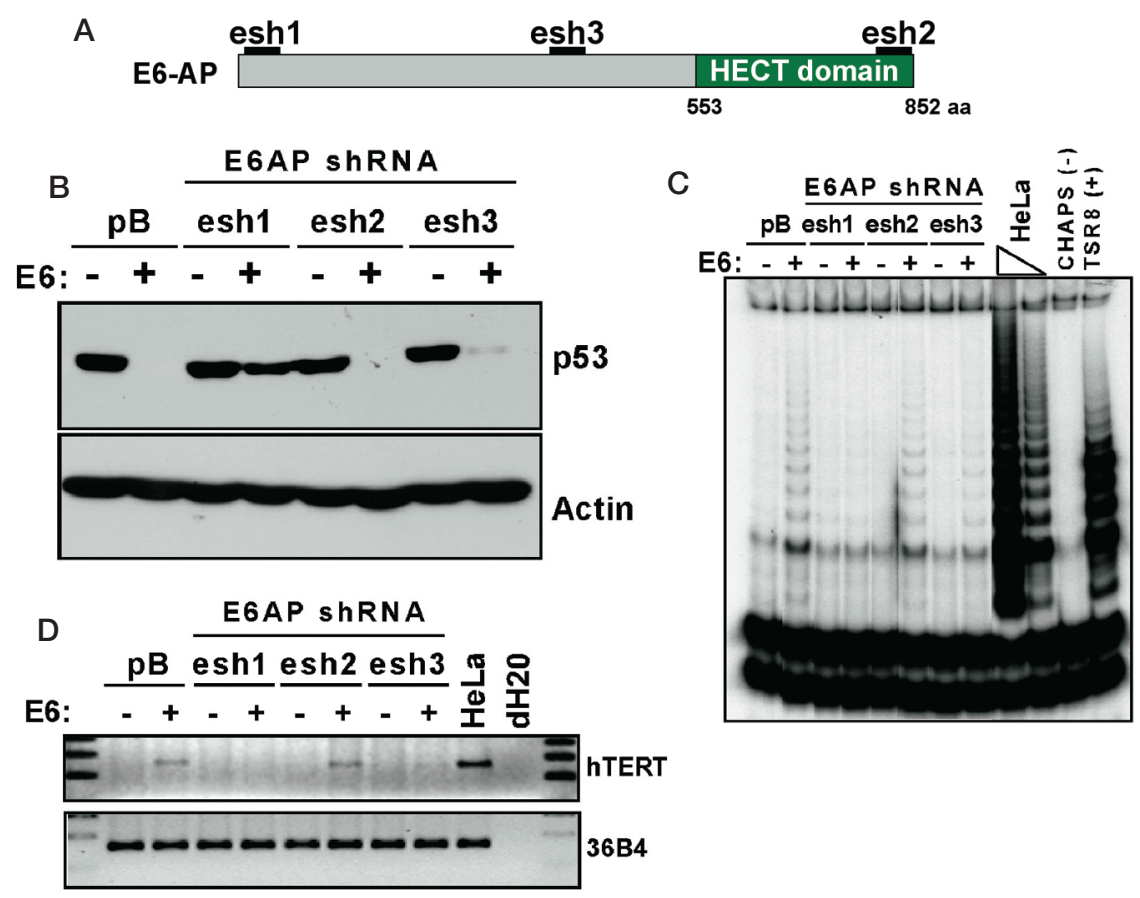

Figure 2. E6AP expression is required for telomerase induction by HPV-16 E6. ( $A$ ) Schematic representation of the three shRNA constructs targeting E6AP (esh1,2,3). (B) HFKs expressing the E6AP shRNA constructs were subsequently transduced with LXSN empty vector $(-)$ or LXSN-16E6 (+). p53 levels were examined by western blot. Actin is a loading control. (C) TRAP assay. Extracts from the same cells shown in $B$ were assayed for telomerase activity. HeLa cells are a positive control lysate. CHAPS is a lysis buffer negative control. TSR8 is a synthetic template of eight telomeric repeats used as a PCR-positive control. (D) RT-PCR. Expression of hTERT RNA was examined by RT-PCR of RNA extracts. $36 B 4$ is a loading control. 


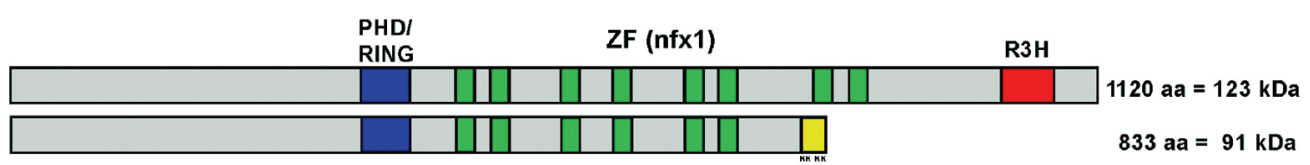

Figure 3. Schematic representation of the two NFX1 isoforms. The blue box represents a PHD/RING finger domain. Multiple green boxes indicate NFX1-type zinc-finger domains. The red box represents an R3H domain present only in NFX1-123. The yellow box indicates the unique lysine-rich carboxy-terminal domain of NFX1-91.

teine/histidine-rich sequences identified as NFX1-type zinc fingers by the Pfam database (www.sanger.ac.uk// cgi-bin/Pfam/getacc?PF01422). Identified as the DNAbinding domain of NFX1 (Song et al. 1994), these zinc fingers $(\mathrm{C}-\mathrm{X}[1-6]-\mathrm{H}-\mathrm{X}-\mathrm{C}-\mathrm{X} 3-\mathrm{C}-[\mathrm{H} / \mathrm{C}]-\mathrm{X}[3-4]-[\mathrm{H} / \mathrm{C}]-$ $\mathrm{X}[1-10]-\mathrm{C})$ are highly homologous to one another but do not exhibit the typical spacing of traditional zinc fingers. NFX1-91 contains the first six zinc fingers followed by a unique lysine-rich stretch of 25 amino acids, whereas NFX1-123 contains eight zinc fingers as well as a region known as an R3H domain believed to be involved in single-stranded nucleic acid binding (Fig. 3). The NFX1 gene is highly conserved among eukaryotic species, although little is known about the functions of its homologs.

In reporter assays using $h T E R T$ promoter constructs that encoded either 219 bp or 710 bp upstream of the transcription start site, NFX1-91 repressed basal transcription or transcription that had been activated by either E6, cMyc, or both (Gewin et al. 2004). Because NFX1 was originally identified in a screen for proteins that bind the $\mathrm{X}$ box of MHC class II genes, the presence of similar sequences in the $h T E R T$ promoter was investigated. The MHC class II X-box sequence is CCTAGCAACAGATG (highly conserved residues are underlined) (Song et al. 1994). Sequence scanning of the promoter found two possible X-box-like sequences within the $h T E R T$ proximal promoter. One of these X-box-like sequences (CGTGGGAAGCCCTG) overlapped with the proximal E box while the other (CCTGGGAACAGGTG) lay in the reverse orientation approximately $400 \mathrm{bp}$ upstream of the transcription start site. To address whether NFX1-91 directly binds the proximal putative $\mathrm{X}$ box within the hTERT promoter, recombinant His-tagged NFX1 protein spanning the zinc fingers and novel carboxy-terminal tail was generated and purified for use in electrophoretic mobility shift assays (EMSA). Using titrations of recombinant protein, we found that His-NFX1-91 bound and shifted a 48-bp region surrounding the proximal E box and overlapping putative $\mathrm{X}$ box, and binding was slightly diminished upon mutation of five residues within the $\mathrm{X}$ box (Gewin et al. 2004).

Although E6 interacted with NFX1-123 in the yeast two-hybrid screen and in in vitro binding assays ( $\mathrm{L}$. Gewin, unpubl.), E6 preferentially bound to NFX1-91 and not to NFX1-123 in co-immunoprecipitations from 293Ts transiently transfected with AU1-tagged E6 (Gewin et al. 2004). This is particularly striking because the steadystate level of NFX1-123 is much greater than that of NFX1-123. Furthermore, the association of E6/E6AP with NFX1-91 resulted in a proteosome-dependent degradation of NFX1-91, which was not seen in HFKs expressing mutated E6 proteins that failed to bind E6AP (Gewin et al. 2004). Preliminary data indicated that degradation of
NFX1-91 was dependent on the four lysine residues in the unique carboxy-terminal tail, because mutation of these residues to alanine resulted in a much more stable protein (L. Gewin and R. Katzenellenbogen, unpubl.). Ubiquitinated NFX1-91 was much more prevalent in HFK/E6 cells than in HFK/LXSN cells, and NFX1-123 did not appear to be ubiquitinated. Longer exposures indicated that there is some ubiquitinated NFX1-91 in HFK/LXSN cells, and ubiquitination of NFX1-91 was readily observed in 293T cells (Gewin et al. 2994). These observations are similar to what is seen with p53; i.e., that p53 stability is regulated by E6/E6AP in E6 cells and by a different ubiquitin ligase, MDM2, in non-E6-expressing cells.

To address directly whether NFX1-91 functions as a transcriptional repressor at the endogenous $h T E R T$ promoter, NFX1-91 expression was reduced using stable shRNA expression in HFKs. We constructed an shRNA construct targeted to the unique $3^{\prime}$ untranslated region (3'UTR) of NFX1-91. The NFX1-91 shRNA (n91sh) reduced NFX1-91 protein levels in HFKs accompanied by derepression of the $h T E R T$ promoter even in cells lacking E6 expression (Gewin et al. 2004). E6 cells with reduced NFX1-91 expression had a greater than twofold increased expression of hTERT as demonstrated by RT-PCR and TRAP assay. Furthermore, in HFK/LXSN cells with reduced NFX1-91 levels, acetylated H3 and H4 were increased at the hTERT promoter in ChIP assays.

Transcriptional repressors often function by recruiting histone deacetylases (HDACs) to the promoter through scaffold proteins such as Sin3A. In vitro co-immunoprecipitation experiments showed that endogenous NFX1-91 directly bound to in vitro translated, ${ }^{35} \mathrm{~S}$-labeled $\mathrm{mSin} 3 \mathrm{~A}$, and in vivo co-immunoprecipitation assays also showed that FLAG-tagged NFX1-91 could bind to myc-tagged mSin3A in 293T cells (W. Luo et al., unpubl.). FLAGtagged-NFX1-91 immunoprecipitates showed high HDAC activity, whereas FLAG-NFX1-123 immunoprecipitates showed relatively lower HDAC activity. Surprisingly, the amino-terminal region of NFX1-91 (upstream of the PHD/RING finger) bound to Sin3A, a region that is in common with NFX1-123. It is unclear why NFX1-91, but not the $123-\mathrm{kD}$ isoform, binds $\operatorname{Sin} 3 \mathrm{~A}$; we speculate that the unique carboxy-terminal tails of the two isoforms influence binding to DNA and to other proteins.

Taken together, our data suggest a model (Fig. 4) in which NFX1-91 functions to repress $h T E R T$ transcription in HFKs by binding to an X-box motif that is located adjacent to the proximal E box in the hTERT promoter. NFX191 binds to $\operatorname{Sin} 3 \mathrm{~A}$ and recruits HDACs to the promoter. It is unclear whether binding of NFX1-91 occludes binding of c-Myc to the proximal E-box. Although we know that cMyc occupies the $h T E R T$ promoter in HFKs, the resolution of the ChIP assay does not allow us to determine whether 


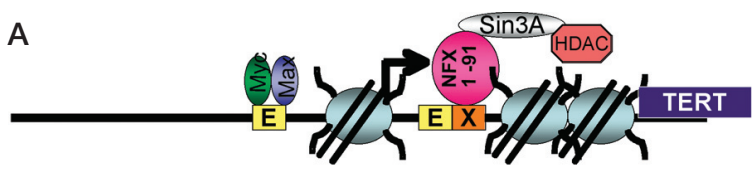

B

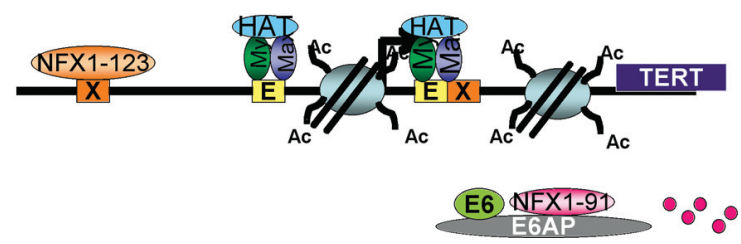

Figure 4. Model for the regulation of the hTERT promoter by HPV-16 E6. (A) In normal HFKs, NFX1-91 binds to an X box that partially overlaps an $\mathrm{E}$ box, perhaps occluding binding of cMyc to the E-box. NFX1-91 binds Sin3A and recruits HDACs to repress transcription. c-Myc is present at the promoter, although unable to activate transcription. $(B)$ In E6-HFKs, NFX191 is targeted for ubiquitin-mediated degradation. This may allow occupancy of the proximal $\mathrm{E}$ box by $\mathrm{c}-\mathrm{Myc}$ and recruitment of HATs. NFX1-123 may bind a distal X-box motif contributing to activation.

c-Myc binds one or both E boxes in the minimal promoter. What is clear is that NFX1-91 binding prevents the recruitment or activity of histone acetyltransferases (HATs) at the hTERT promoter. In E6-expressing HFKs, NFX1-91 binds to the E6/E6AP complex, and it is ubiquitinated and targeted for proteosomal degradation. It is possible that simply removing NFX1-91 from the promoter is sufficient to allow c-Myc to activate transcription of hTERT; alternatively, this may be only one step in the process of robustly inducing hTERT expression by E6. Given that E6/E6AP and the NFX1 RING finger possess E3 ubiquitin ligase activity, it is possible that other proteins at the promoter, such as histone $\mathrm{H} 2 \mathrm{~B}$, c-Myc, or other transcriptional regulators, may be ubiquitinated by bringing E6/E6AP to the promoter. An attractive synthesis of this model with our data is that E6/E6AP may function at multiple levels to induce hTERT. First, the E6/E6AP complex may target NFX1-91 for increased turnover to derepress the promoter; then, an E3 ligase may ubiquitinate and activate the c-Myc bound at the hTERT promoter. This theory and the possible role of the E3 ligase activity of the NFX1 isoforms or the E6/E6AP complex to ubiquitinate either histone $\mathrm{H} 2 \mathrm{~B}$ or cMyc remain to be tested.

\section{NFX1-123 AND ITS ROLE IN hTERT TRANSCRIPTION}

Reporter assays indicated that NFX1-123 could co-activate the $h T E R T$ promoter when present with c-Myc, E6, or both. We considered a simple model in which degradation of NFX1-91 by E6/E6AP would lead to replacement with NFX1-123 at the proximal E box and recruitment of histone acetyltransferases, but that model seems incorrect for several reasons. First, NFX1-123 did not activate transcription in promoter assays when only the 219-bp promoter was used, nor did it bind to the proximal $\mathrm{X}$ box in gel-shift assays. Second, NFX1-123 is much more abundant and stable than NFX1-91; thus, one might anticipate that if NX1 could occupy the proximal X box, it would displace NFX1-91. A second X box is located about 400 bp upstream of the proximal $\mathrm{X}$ box, and we will examine binding of NFX1-123 to that site.

As another approach to investigate how NFX1-123 might regulate transcription of hTERT, we used tagged forms of NFX1-123 and identified co-immunoprecipitating proteins by mass spectrometry (R.A. Katzenellenbogen et al., unpubl.). Of interest, several poly(A)-binding proteins were identified. Cytoplasmic poly(A)-binding proteins (PABPCs) contain four RNA recognition motifs (RRM) in their amino terminus, which define the fidelity with which they bind poly(A) or poly(AU) RNA (Kuhn and Wahle 2004). At their carboxyl termini, PABPCs have a poly(A)-binding protein carboxy-terminal domain, similarly named PABC, and with this domain they interact with other proteins that have a poly(A)-binding interacting motif, named PAM2. Cytoplasmic poly(A)binding proteins bind to the $3^{\prime}$ poly(A) tail of mRNA and directly interact with $5^{\prime}$ cap proteins, forming a closed loop of RNA. This loop helps recruit the $40 \mathrm{~S}$ ribosome subunit to the mRNA and may in fact keep ribosomes on the same mRNA, thereby allowing reinitiation of translation. These proteins also stabilize mRNA transcripts by blocking premature decapping of the mRNA and by preventing deadenylation of mRNA. Thus, PABPs work downstream of transcriptional activation; they lengthen the half-life of mRNA and increase protein expression through improved translation. We found that NFX1-123 has a putative PAM2 motif in its amino terminus. In preliminary experiments, we found that NFX1-123 can interact with poly(A)-binding proteins and that mutation of the PAM2 site significantly reduces that interaction. Importantly, mutation of that site reduced expression of luciferase in the hTERT reporter assays. Thus, we are exploring a model (Fig. 5) in which NFX1-123 may augment hTERT expression by a posttranscriptional mechanism. This mechanism may act in concert with direct activation of the $h T E R T$ promoter through binding of NFX1-123 to the distal X box in the hTERT promoter.

\section{ACTIVATION OF TELOMERASE AND THE PAPILLOMAVIRUS LIFE CYCLE}

Viruses, particularly those with small genomes, likely only retain gene products that in some way benefit their life cycle. HPVs infect basal cells in cutaneous or mucosal

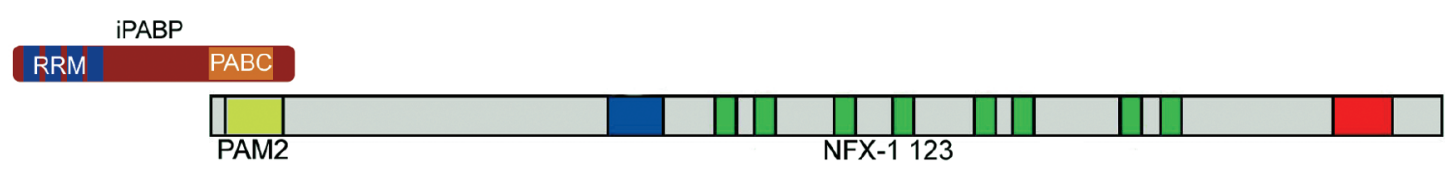

Figure 5. Schematic of interactions between poly(A)-binding proteins with NFX1-123. A PABPC motif in the carboxyl terminus of the inducible poly(A)-binding protein (iPABP) interacts with the PAM2 domain in the amino terminus of NFX1-123. 
epithelium and complete their life cycle entirely within the epithelium. HPVs induce cellular replication of otherwise quiescent cells, expanding the proliferative compartment of the epithelial layer, yet epithelial differentiation proceeds and is required for the expression of the capsid proteins (Howley and Schlegel 1988). Overall, the number of population doublings that an HPV-infected epithelial cell will undergo are likely to be too few to critically shorten telomeres. Therefore, we have wondered how induction of telomerase benefits human papillomaviruses and have considered three possible explanations.

First, HPV likely remains latent or persistent in epithelial cells, and the induction of telomerase might confer long-lasting replicative potential. Although this is possible, there is no evidence that cells which harbor HPV undergo extensive replication. Additionally, HPV may remain latent in stem cells in which telomerase is constitutively active.

A second possibility is that NFX1-91 likely represses many genes in addition to $h T E R T$, and derepression of some of these other genes by E6/E6AP may be important for the virus life cycle. We are currently identifying other targets of NFX1.

A third possibility is that telomerase has activities other than elongation of telomeres which may be beneficial to HPVs. Several lines of evidence lend support to this hypothesis. hTERT has been shown to be required for tumorigenic transformation of cells that are able to maintain their telomeres by the ALT mechanism (Stewart et al. 2002). Expression of hTERT in two different cell types resulted in increased expression of genes in the EGFR signaling pathway (Lindvall et al. 2003; Smith et al. 2003), implying that hTERT might stimulate growth. Induction of TERT expression in mouse skin lacking the RNA component of telomerase resulted in the proliferation of normally quiescent stem cells in hair follicles (Sarin et al. 2005). Additionally, we reported that expression of hTERT in young fibroblasts with long telomeres increased the density at which the cells could grow and strongly cooperated with activated Ha-Ras to increase saturation density (Benanti and Galloway 2004). Thus, we speculate that the induction of telomerase by human papillomaviruses might provide a growth advantage to HPV-infected cells that is independent of telomere lengthening.

\section{CONCLUSIONS}

Induction of telomerase appears to be a common and requisite event in the immortalization and transformation of many cell types, and in the development of human cancers. In our studies to determine how HPV-16 E6 induces telomerase in epithelial cells, we have identified NFX191 as a cellular repressor of the human hTERT promoter both in vivo and in vitro that is destabilized by the E6/E6AP complex. A related splice variant, NFX1-123, can co-activate the $h T E R T$ promoter, although its role in telomerase regulation remains to be defined.

A recent study found three different tumor suppressor/oncogene pathways involved in hTERT repression (Lin and Elledge 2003). It is striking that these repressors do not seem redundant; oncogenic stimulation that abrogates any one of them is sufficient to relieve repression. Therefore, it is not implausible that, despite the identification of several hTERT repressor proteins, E6/E6AP specifically targets only one transcriptional repressor, NFX1-91, to induce hTERT expression. It is likely that future investigations will reveal different cell-type specificities for $h T E R T$ repressors and variability in the responsiveness of different cell types to perturbations of these repressors. How, or whether, induction of hTERT benefits HPV-infected cells is speculative but may involve telomere-independent activities of hTERT.

\section{ACKNOWLEDGMENTS}

We thank members of the Galloway and McDougall labs, past and present, for their many contributions to this work, particularly Al Klingelhutz, Scott Foster, Tohru Kiyono, Jenn Koop, and Jenny Benanti. This work was supported by grants from the National Cancer Institute (CA42792 and CA64795) to D.A.G. and J.K.M. L.C.G. was supported in part by the Viral Oncology Training grant (T32 CA 09229-23), and R.A.K. and L.C.G. were supported in part by a STD/AIDS Research Training grant (T32 AI07140).

\section{REFERENCES}

Benanti J.A. and Galloway D.A. 2004. Normal human fibroblasts are resistant to RAS-induced senescence. Mol. Cell. Biol. 24: 2842.

Bodnar A.G., Ouellette M., Frolkis M., Holt S.E., Chiu C.P., Morin G.B., Harley C.B., Shay J.W., Lichtsteiner S., and Wright W.E. 1998. Extension of life-span by introduction of telomerase into normal human cells (comment). Science 279: 349.

Cogliano V., Baan R., Straif K., Grosse Y., Secretan B., El Ghissassi F., and the W.H.O. International Agency for Research on Cancer. 2005. Carcinogenicity of human papillomaviruses. Lancet Oncol. 6: 204.

Demers G.W., Foster S.A., Halbert C.L., and Galloway D.A. 1994. Growth arrest by induction of p53 in DNA damaged keratinocytes is bypassed by human papillomavirus 16 E7. Proc. Natl. Acad. Sci. 91: 4382.

Ducrest A.L., Amacker M., Mathieu Y.D., Cuthbert A.P., Trott D.A., Newbold R.F., Nabholz M., and Lingner J. 2001. Regulation of human telomerase activity: Repression by normal chromosome 3 abolishes nuclear telomerase reverse transcriptase transcripts but does not affect c-Myc activity. Cancer Res. 61: 7594.

Gewin L. and Galloway D.A. 2001. E box-dependent activation of telomerase by human papillomavirus type 16 E6 does not require induction of c-myc. J. Virol. 75: 7198.

Gewin L., Myers H., Kiyono T., and Galloway D.A. 2004. Identification of a novel telomerase repressor that interacts with the human papillomavirus type-16 E6/E6-AP complex. Genes Dev. 18: 2269.

Greenberg R.A., O’Hagan R.C., Deng H., Xiao Q., Hann S.R., Adams R.R., Lichtsteiner S., Chin L., Morin G.B., and DePinho R.A. 1999. Telomerase reverse transcriptase gene is a direct target of c-Myc but is not functionally equivalent in cellular transformation. Oncogene 18: 1219.

Gross-Mesilaty S., Reinstein E., Bercovich B., Tobias K.E., Schwartz A.L., Kahana C., and Ciechanover A. 1998. Basal and human papillomavirus E6 oncoprotein-induced degradation of Myc proteins by the ubiquitin pathway. Proc. Natl. Acad. Sci. 5: 8058. 
Hahn W.C., Counter C.M., Lundberg A.S., Beijersbergen R.L., Brooks M.W., and Weinberg R.A. 1999. Creation of human tumour cells with defined genetic elements (comment). $\mathrm{Na}$ ture 400: 464.

Helt A.M. and Galloway D.A. 2001. Destabilization of the retinoblastoma tumor suppressor by human papillomavirus type $16 \mathrm{E} 7$ is not sufficient to overcome cell cycle arrest in human keratinocytes. J. Virol. 75: 6737.

Horikawa I. and Barrett J.C. 2003. Transcriptional regulation of the telomerase hTERT gene as a target for cellular and viral oncogenic mechanisms. Carcinogenesis 24: 1167.

Horikawa I., Oshimura M., and Barrett J.C. 1998. Repression of the telomerase catalytic subunit by a gene on human chromosome 3 that induces cellular senescence. Mol. Carcinog. 22: 65.

Hou M., Wang X., Popov N., Zhang A., Zhao X., Zhou R., Zetterberg A., Bjorkholm M., Henriksson M., Gruber A., and $\mathrm{Xu}$ D. 2002. The histone deacetylase inhibitor trichostatin A derepresses the telomerase reverse transcriptase (hTERT) gene in human cells. Exp. Cell Res. 274: 25.

Howley P.M. and Schlegel R. 1988. The human papillomaviruses: An overview. Am. J. Med. 85: 155.

Huibregtse J.M., Scheffner M., and Howley P.M. 1993. Localization of the E6-AP regions that direct human papillomavirus E6 binding, association with p53, and ubiquitination of associated proteins. Mol. Cell. Biol. 13: 4918.

Kessis T.D., Slebos R.J., Nelson W.G., Kastan M.B., Plunkett B.S., Han S.M., Lorincz A.T., Hedrick L., and Cho K.R. 1993. Human papillomavirus 16 E6 expression disrupts the p53-mediated cellular response to DNA damage. Proc. Natl. Acad. Sci. 90: 3988.

Kim N.W., Piatyszek M.A., Prowse K.R., Harley C.B., West M.D., Ho P.L.C., Coviello G.M., Wright W.E., Weinrich S.L., and Shay J.W. 1994. Specific association of human telomerase activity with immortal cells and cancer. Science 266: 2011.

Kinoshita T., Shirasawa H., Shino Y., Moriya H., Desbarats L., Eilers M., and Simizu B. 1997. Transactivation of prothymosin $\alpha$ and c-myc promoters by human papillomavirus type 16 E6 protein. Virology 232: 53.

Kiyono T., Foster S.A., Koop J.I., McDougall J.K., Galloway D.A., and Klingelhutz A.J. 1998. Both Rb/p16INK4a inactivation and telomerase activity are required to immortalize human epithelial cells (comment). Nature 396: 84.

Klingelhutz A.J., Foster S.A., and McDougall J.K. 1996. Telomerase activation by the E6 gene product of human papillomavirus type 16. Nature 380: 79 .

Klingelhutz A.J., Barber S.A., Smith P.P., Dyer K., and McDougall J.K. 1994. Restoration of telomeres in human papillomavirus-immortalized human anogenital epithelial cells. Mol. Cell. Biol. 14: 961.

Kuhn U. and Wahle E. 2004. Structure and function of poly(A) binding proteins. Biochim. Biophys. Acta 1678: 67.

Lin S.Y. and Elledge S.J. 2003. Multiple tumor suppressor pathways negatively regulate telomerase. Cell 113: 881.

Lindvall C., Hou M., Komurasaki T., Zheng C., Henriksson M., Sedivy J.M., Bjorkholm M., Teh B.T., Nordenskjold M., and $\mathrm{Xu}$ D. 2003. Molecular characterization of human telomerase reverse transcriptase-immortalized human fibroblasts by gene expression profiling: Activation of the epiregulin gene. Cancer Res. 63: 1743.

Lorick K.L., Jensen J.P., Fang S., Ong A.M., Hatakeyama S., and Weissman A.M. 1999. RING fingers mediate ubiquitinconjugating enzyme (E2)-dependent ubiquitination. Proc. Natl. Acad. Sci. 96: 11364.

McDougall J.K. 1994. Immortalization and transformation of human cells by human papillomavirus. Curr. Top. Microbiol. Immunol. 186: 101 .

Munger K., Basile J.R., Duensing S., Eichten A., Gonzalez S.L., Grace M., and Zacny V.L. 2001. Biological activities and molecular targets of the human papillomavirus E7 oncoprotein. Oncogene 20: 7888.

Oh S.T., Kyo S., and Laimins L.A. 2001. Telomerase activation by human papillomavirus type $16 \mathrm{E} 6$ protein: Induction of human telomerase reverse transcriptase expression through Myc and GC-rich Sp1 binding sites. J. Virol. 75: 5559.

Sarin K.Y., Cheung P., Gilison D., Lee E., Tennen R.I., Wang E., Artandi M.K., Oro A.E., and Artandi S.E. 2005. Conditional telomerase induction causes proliferation of hair follicle stem cells (comment). Nature 436: 1048.

Shay J.W., Wright W.E., and Werbin H. 1991. Defining the molecular mechanisms of human cell immortalization. Biochim. Biophys. Acta 1072: 1

Smith L.L., Coller H.A., and Roberts J.M. 2003. Telomerase modulates expression of growth-controlling genes and enhances cell proliferation. Nat. Cell Biol. 5: 474.

Song Z., Krishna S., Thanos D., Strominger J.L., and Ono S.J. 1994. A novel cysteine-rich sequence-specific DNA-binding protein interacts with the conserved X-box motif of the human major histocompatibility complex class II genes via a repeated Cys-His domain and functions as a transcriptional repressor. J. Exp. Med. 180: 1763.

Stewart S.A., Hahn W.C., O'Connor B.F., Banner E.N., Lundberg A.S., Modha P., Mizuno H., Brooks M.W., Fleming M., Zimonjic D.B., Popescu N.C., and Weinberg R.A. 2002. Telomerase contributes to tumorigenesis by a telomere length-independent mechanism (comment). Proc. Natl. Acad. Sci. 99: 12606.

Takakura M., Kyo S., Sowa Y., Wang Z., Yatabe N., Maida Y., Tanaka M., and Inoue M. 2001. Telomerase activation by histone deacetylase inhibitor in normal cells. Nucleic Acids Res. 29: 3006.

van Steensel B., Smogorzewska A., and deLange T. 1998. TRF2 protects human telomeres from end-to-end fusions. Cell 92: 401.

Vaziri H. and Benchimol S. 1998. Reconstitution of telomerase activity in normal human cells leads to elongation of telomeres and extended replicative life span. Curr. Biol. 8: 279.

Veldman T., Horikawa I., Barrett J.C., and Schlegel R. 2001. Transcriptional activation of the telomerase hTERT gene by human papillomavirus type 16 E6 oncoprotein. J. Virol. 75: 4467.

Veldman T., Liu X., Yuan H., and Schlegel R. 2003. Human papillomavirus E6 and Myc proteins associate in vivo and bind to and cooperatively activate the telomerase reverse transcriptase promoter. Proc. Natl. Acad. Sci. 100: 8211.

Walboomers J.M., Jacobs M.V., Manos M.M., Bosch F.X., Kummer J.A., Shah K.V., Snijders P.J., Peto J., Meijer C.J., and Munoz N. 1999. Human papillomavirus is a necessary cause of invasive cervical cancer worldwide (comment). $J$. Pathol. 189: 12 .

Wang J., Xie L.Y., Allan S., Beach D., and Hannon G.J. 1998. Myc activates telomerase. Genes Dev. 12: 1769.

Wu K.-J., Grandori C., Amacker M., Simon-Vermot N., Polack A., Lingner J., and Dalla-Favera R. 1999. Direct activation of TERT transcription by c-MYC. Nat. Genet. 21: 220.

Xu D., Popov N., Hou M., Wang Q., Bjorkholm M., Gruber A., Menkel A.R., and Henriksson M. 2001. Switch from Myc/Max to Mad1/Max binding and decrease in histone acetylation at the telomerase reverse transcriptase promoter during differentiation of HL60 cells. Proc. Natl. Acad. Sci. 98: 3826 . 


\section{$\$_{\text {CSH\& }}^{\infty} \mathrm{Cold}$ Spring Harbor Symposia SYMPOSIA on Quantitative Biology}

\section{Regulation of Telomerase by Human Papillomaviruses}

D.A. GALLOWAY, L.C. GEWIN, H. MYERS, et al.

Cold Spring Harb Symp Quant Biol 2005 70: 209-215

Access the most recent version at doi:10.1101/sqb.2005.70.041

References This article cites 43 articles, 20 of which can be accessed free at: http://symposium.cshlp.org/content/70/209.full.html\#ref-list-1

\section{License}

Email Alerting Receive free email alerts when new articles cite this article - sign up in Service the box at the top right corner of the article or click here. 